\title{
Transatlantica
}

Revue d'études américaines. American Studies Journal

\section{La loi « Don't Ask, Don't Tell » vue par deux photographes américains : Jeff Sheng et Antony Loverde}

\section{Clément Massé}

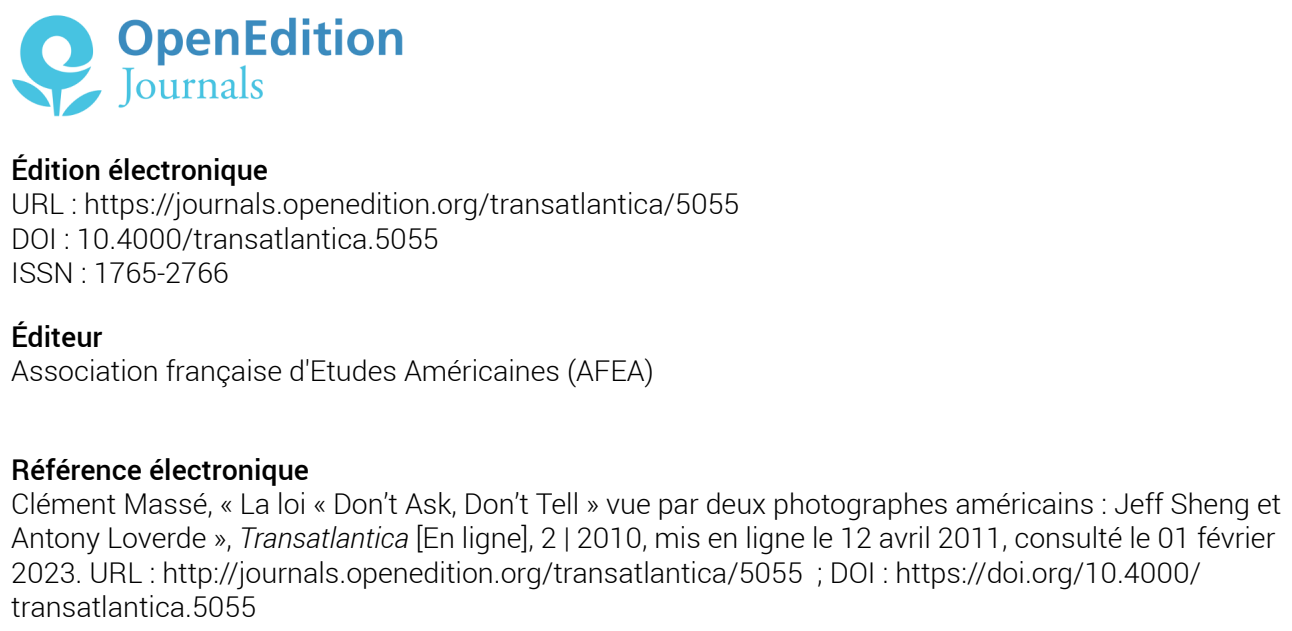

Ce document a été généré automatiquement le 1 février 2023.

\section{(c) $(1) \Theta \Theta$}

Creative Commons - Attribution - Pas d'Utilisation Commerciale - Pas de Modification 4.0 International - CC BY-NC-ND 4.0

https://creativecommons.org/licenses/by-nc-nd/4.0/ 


\title{
La loi « Don't Ask, Don't Tell » vue par deux photographes américains : Jeff Sheng et Antony Loverde
}

\author{
Clément Massé
}

Avec la loi «Don't Ask, Don't Tell », la photographie américaine s'est emparée d'un nouveau sujet. Signée par Bill Clinton en 1993 et abrogée par Barack Obama le 22 décembre 2010, cette loi a interdit pendant dix-sept ans à toute personne servant dans l'armée américaine d'afficher son orientation homosexuelle, bisexuelle ou transsexuelle. Le dire, c'était l'assurance d'être démobilisé et renvoyé, quels que soient les états de service du soldat visé.

En 2010, dans les mois qui ont précédé l'abrogation du texte, les photographes Jeff Sheng et Anthony Loverde ont chacun présenté leur vision, l'un en couleurs, l'autre en noir et blanc, des conséquences de l'application de cette loi.

\section{Jeff Sheng}

Jeff Sheng est connu au États-Unis pour sa précédente série photographique intitulée "Fearless", sur des athlètes lycéens revendiquant leur identité sexuelle, lesbienne, gay, bisexuelle ou transsexuelle1. Sa série de photographies intitulée « Don't Ask, Don't Tell » (qui fait l'objet de deux livres aux États-Unis) retrace le vécu d'une soixantaine de membres des forces armées américaines, hommes et femmes, affectés par cette loi ${ }^{2}$. Au moment où ils ont été photographiés, tous étaient dans les rangs : ils ne pouvaient donc pas révéler leur homosexualité. Les images de Jeff sheng présentent des soldats dont on ne voit pas le visage ou dont on ne distingue que quelques traits : une silhouette, un reflet dans un écran de télévision, un profil. 
1. « Mike, Boston, Massachusetts, 2009 »

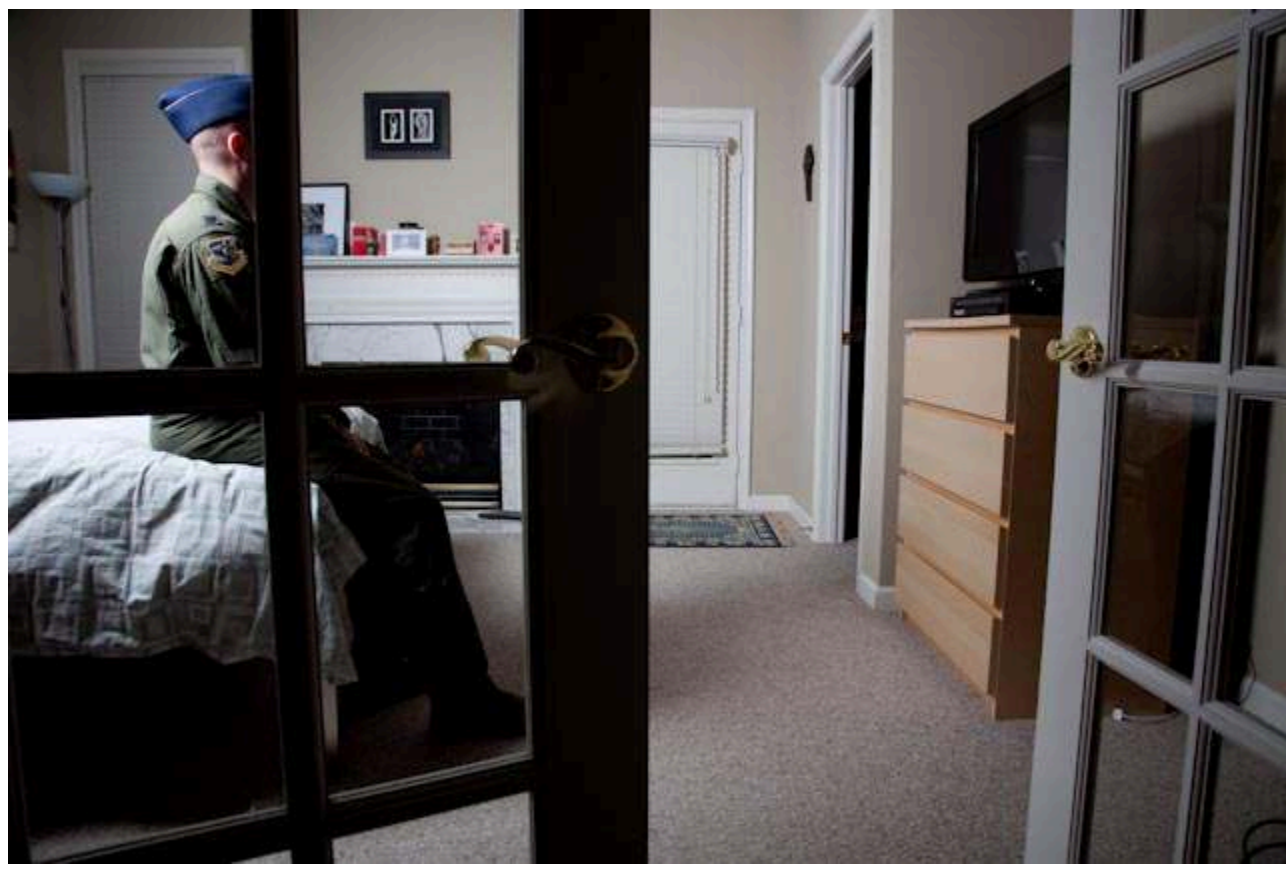

Le visage des sujets de Jeff Sheng est caché par une ombre portée ou coupé par les lignes et les angles du cadre, des cadres dans le cadre (comme dans la photographie cidessus, intitulée « Mike, Boston, Massachusetts, 2009 ») ou encore sciemment dissimulé par le soldat lui-même qui a choisi le degré d'anonymat qu'il souhaitait maintenir, comme dans l'image ci-dessous, intitulée « Jess, Bend, Oregon, 2009 ».

2. « Jess, Bend, Oregon, 2009 »

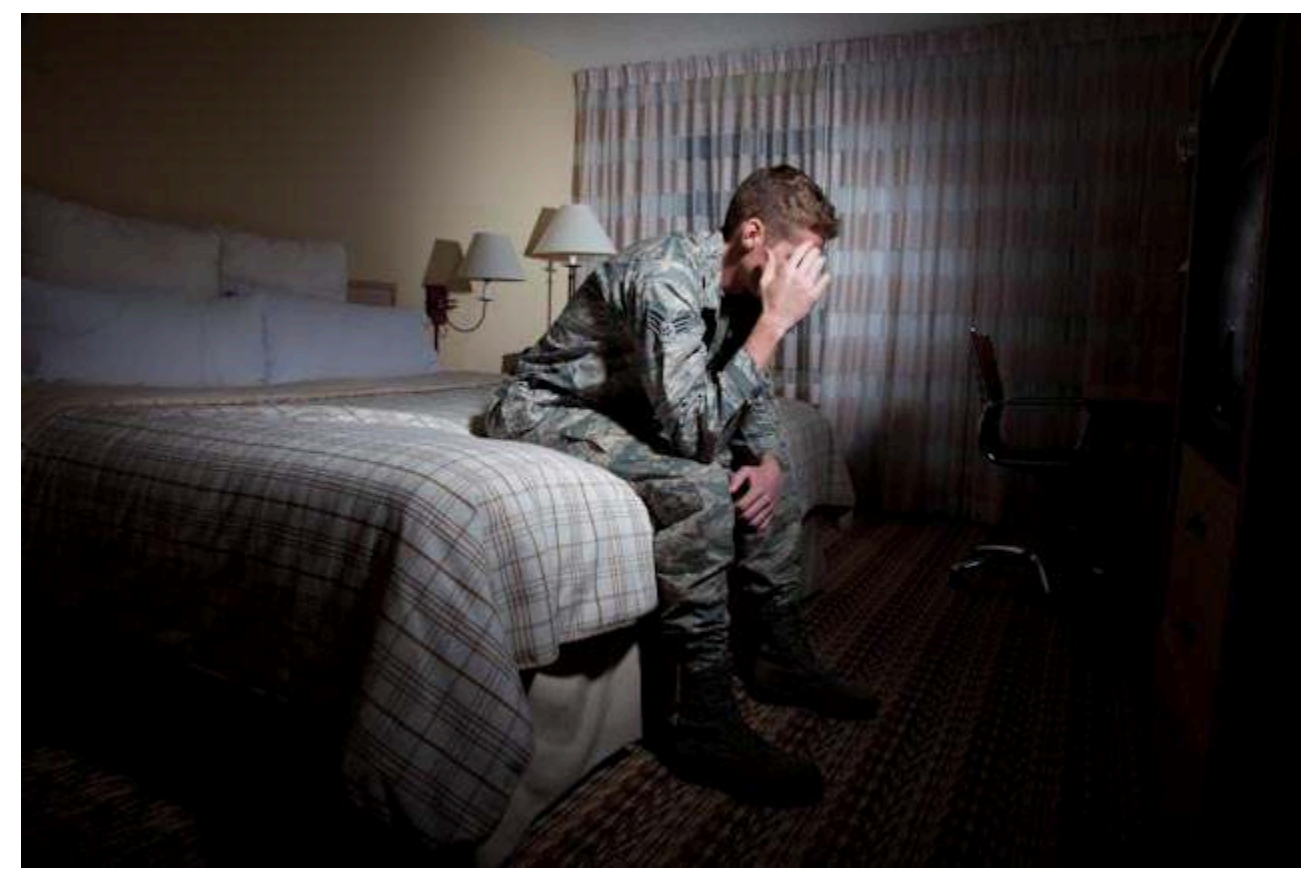

Autre exemple: « Grace, Fort Jackson, South Carolina, 2010 » 
3. « Grace, Fort Jackson, South Carolina, 2010 »

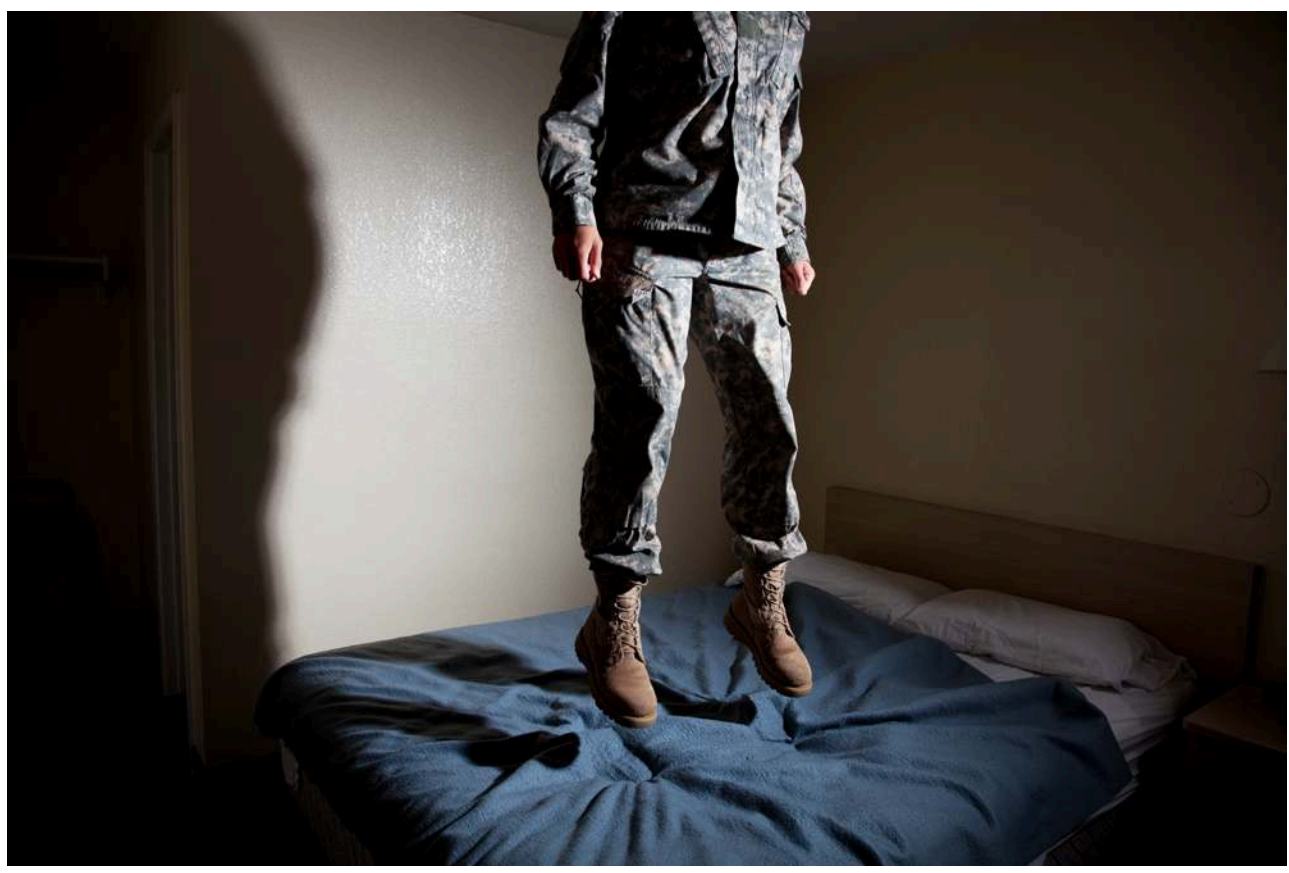

Cette photographie de Jeff Sheng compte parmi les plus saisissantes présentées à la Kaycee Olsen Gallery de Los Angeles, du 18 septembre au 23 octobre 2010. La première impression est celle d'un corps pendu, tant l'image semble être la représentation d'un suicide. La tête est coupée, hors cadre, les pieds sont ballants et leur ombre sur la couverture et celle, portée, sur le mur semblent là pour appuyer la noirceur du drame qui s'est déroulé. Mais on décèle une énergie dans ce corps (celui d'une jeune femme prénommée Grace), et on comprend qu'il est finalement juste en suspens et que Grace a été photographiée alors qu'elle faisait des bonds sur son lit. Il n'en reste pas moins qu'elle incarne ici une sorte de sujet décapité par l'application du texte de loi. Seule reste visible son identité militaire, représentée par son uniforme. 
4. « Katherine \& Elizabeth, Denver, Colorado, 2010 »

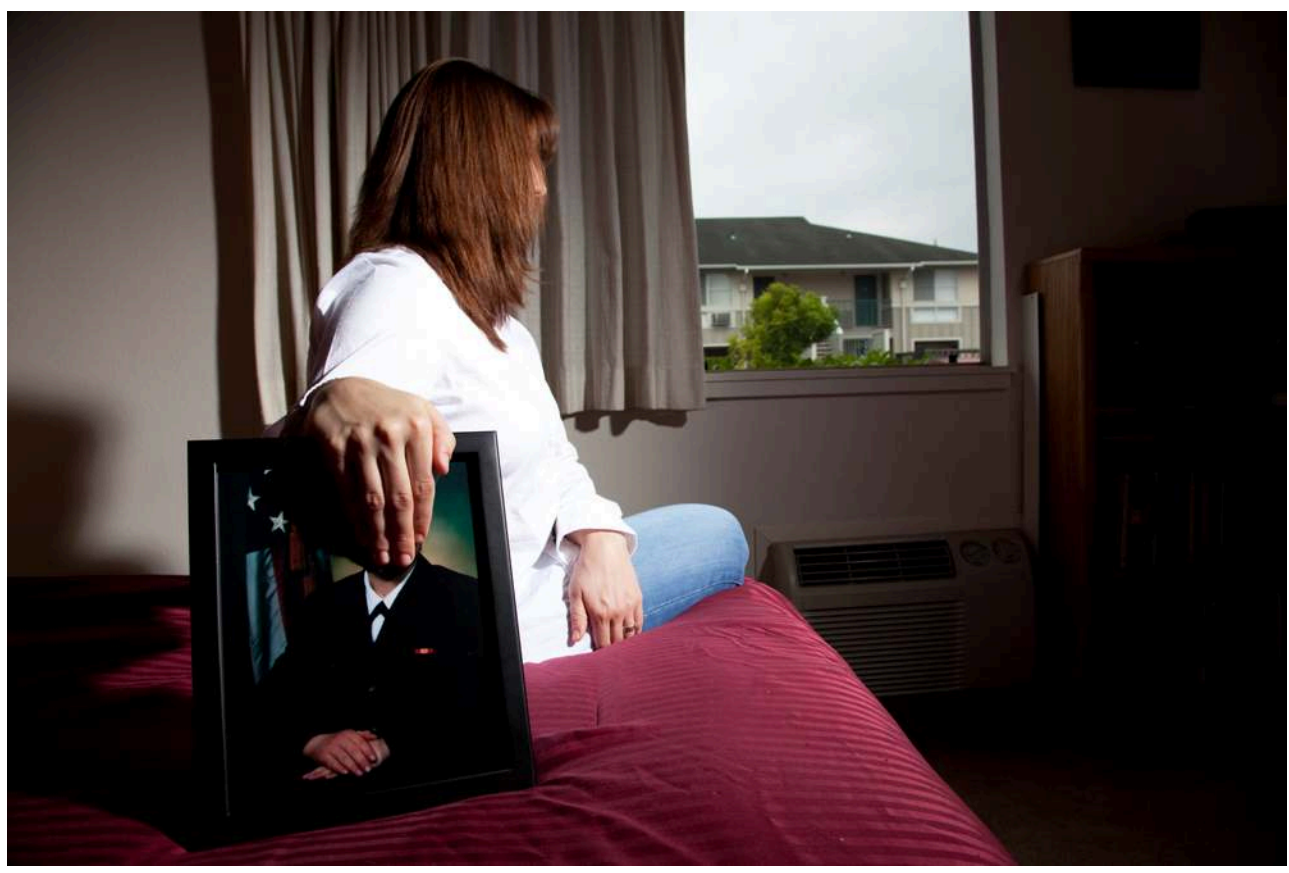

Les sujets de Jeff sheng se définissent eux-mêmes avant tout comme militaires et sont systématiquement photographiés en tenue. L'un des rares clichés où l'uniforme apparaît moins ostensiblement est celui de cette femme dont le visage est voilé à la fois par ses cheveux (donc, invisible à l'objectif du photographe) et par le rideau à moitié tiré (donc, invisible aux regards extérieurs) et qui soutient le cadre d'une photographie où figure sa compagne, dont elle dissimule le visage de la main. Cette image semble suggérer que par extension, les compagnes et compagnons de personnels militaires gays sont eux aussi contraints de vivre cachés pour ne pas risquer de violer la loi et compromettre la carrière de leur conjoint. Ce n'est plus une, mais deux identités qui sont bafouées. La photographie met en scène la négation de l'être. À chaque fois, le tabou de l'homosexualité force à tirer un voile : la main sur le cadre, les cheveux sur le visage, le rideau tiré sur la scène photographiée. Ce qui reste visible par la fenêtre, c'est le petit immeuble d'habitation de l'autre côté de la rue où l'on imagine que se déroulent des vies conformes à la vision jugée acceptable par l'institution militaire.

Par ailleurs, les photographies de Jeff Sheng présentent souvent des sujets figés comme s'ils se retrouvaient aux arrêts, ce qui est le cas de la photographie suivante : 


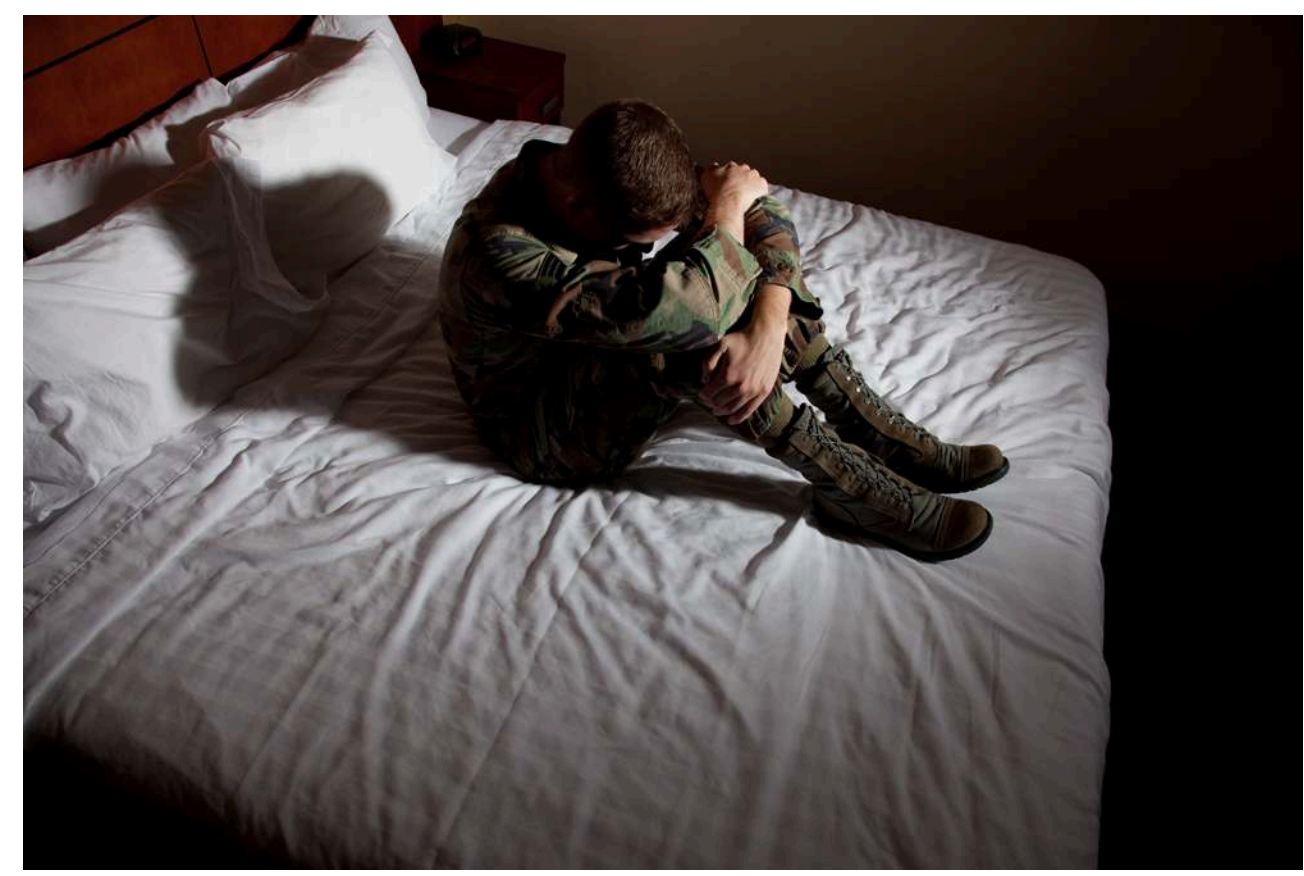

Sur cette photographie, Dean semble être un jeune homme, sans doute tout juste âgé de dix-huit ans. La posture qu'il adopte, recroquevillé sur lui-même, la tête cachée entre ses bras, le protège de la vive lumière blanche du projecteur braqué sur lui. Il est présenté comme un enfant humilié ou puni ou comme une personne déterminée à ne rien trahir pendant une interrogation, dénonçant ainsi le quotidien du personnel militaire homosexuel aux États-Unis. Piégé par l'application d'une loi qui le force à mentir aux autres sur lui-même, le jeune homme est condamné à garder son secret, blotti dans la solitude d'une chambre. Le caractère intime de l'image, mettant en évidence le motif du lit, n'est pas sans évoquer l'ambiance de la photographie de Nan Goldin, prompte à représenter et à dévoiler la sexualité de ses sujets, à cette différence près que sur les images de Jeff Sheng, l'intimité sexuelle ne peut s'exprimer. Elle est contrainte au refoulement, d'où l'expression d'une forme de douleur que l'on devine dans la pose du sujet.

Les images de Sheng expriment ainsi le conflit intérieur du sujet photographié, mais aussi celui d'une armée face à l'évolution de la société. Si la personne se plie, contrainte et forcée, au règlement imposé, la transgression consiste à poser devant l'objectif, afin de mettre l'institution militaire face à l'humiliation qu'elle lui impose.

\section{Anthony Loverde}

Sur ce même sujet, le travail d'Anthony Loverde, dont les photographies présentent aussi des visages cachés, relève d'une démarche souvent plus revendicative.

The Silent Force: Men and Women Serving Under Don't Ask, Don't Tell est son premier livre de photographies ${ }^{3}$. La « Silent Force » du titre, c'est cette partie du personnel militaire, homosexuelle, réduite au silence. Ce jeune photographe a fait carrière dans l'armée avant d'en être remercié lorsqu'il a révélé à ses supérieurs qu'il était homosexuel. Il a notamment servi en Irak. Après son exclusion des rangs, il a rejoint un prestataire privé de l'armée américaine qui l'a renvoyé en Irak exercer des fonctions similaires. Il 
s'est également engagé dans un Master's of Fine Arts à l'Academy of Art (Université de San Francisco) pour lequel il a réalisé les photographies présentées dans son livre.

Son livre se présente comme "un moyen pour mes collègues d'avoir voix au chapitre dans le débat national » (sur l'abrogation de la loi « Don't Ask, Don't Tell ») ${ }^{4}$. Au cœur de son récit photographique se trouvent les valeurs de l'institution militaire qu'il a longtemps représentée en portant l'uniforme : « Devoir, Honneur et Dévouement », ce qu'expriment les photographies suivantes.

\section{1. «A Silent Force, Integrity »}

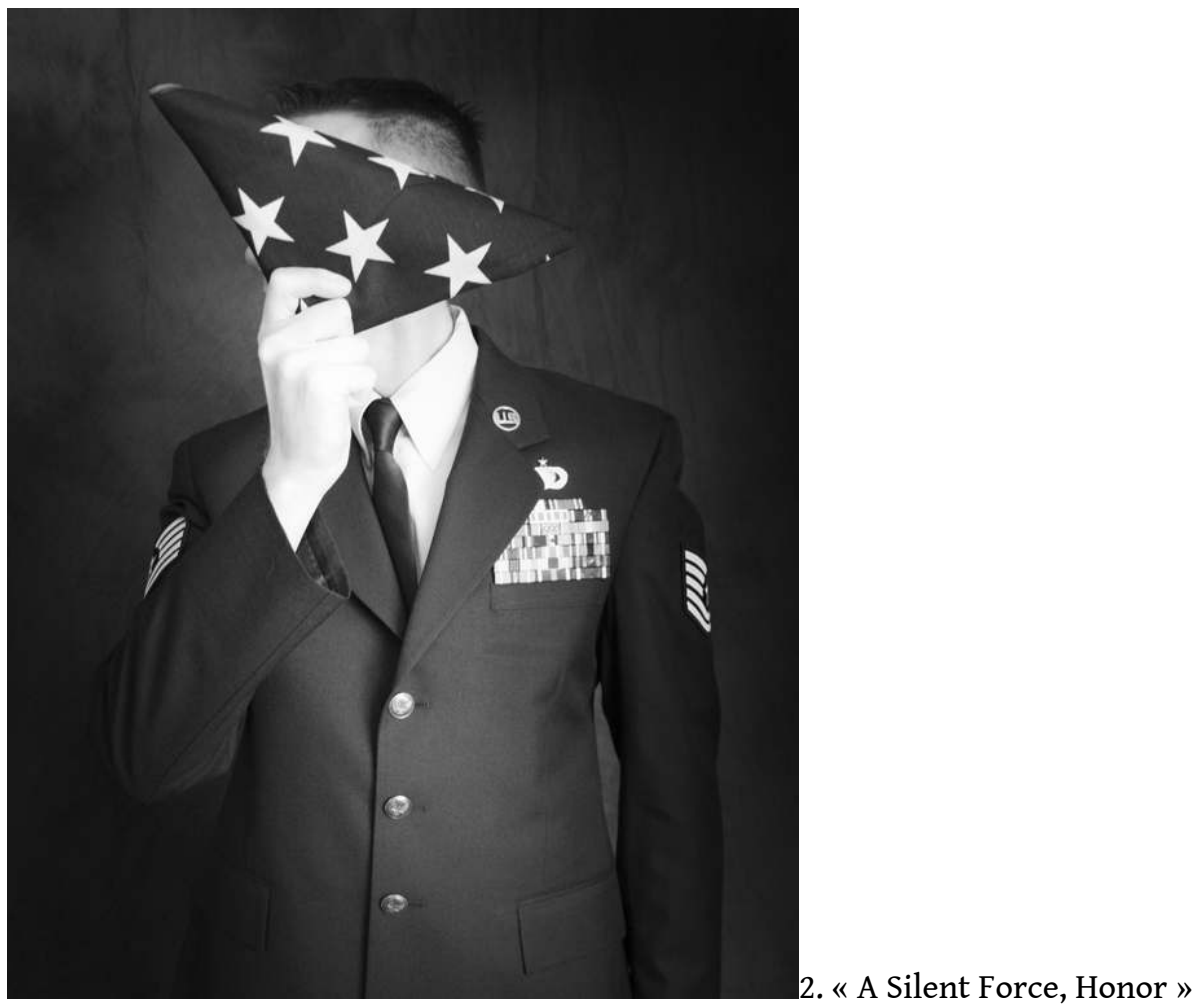



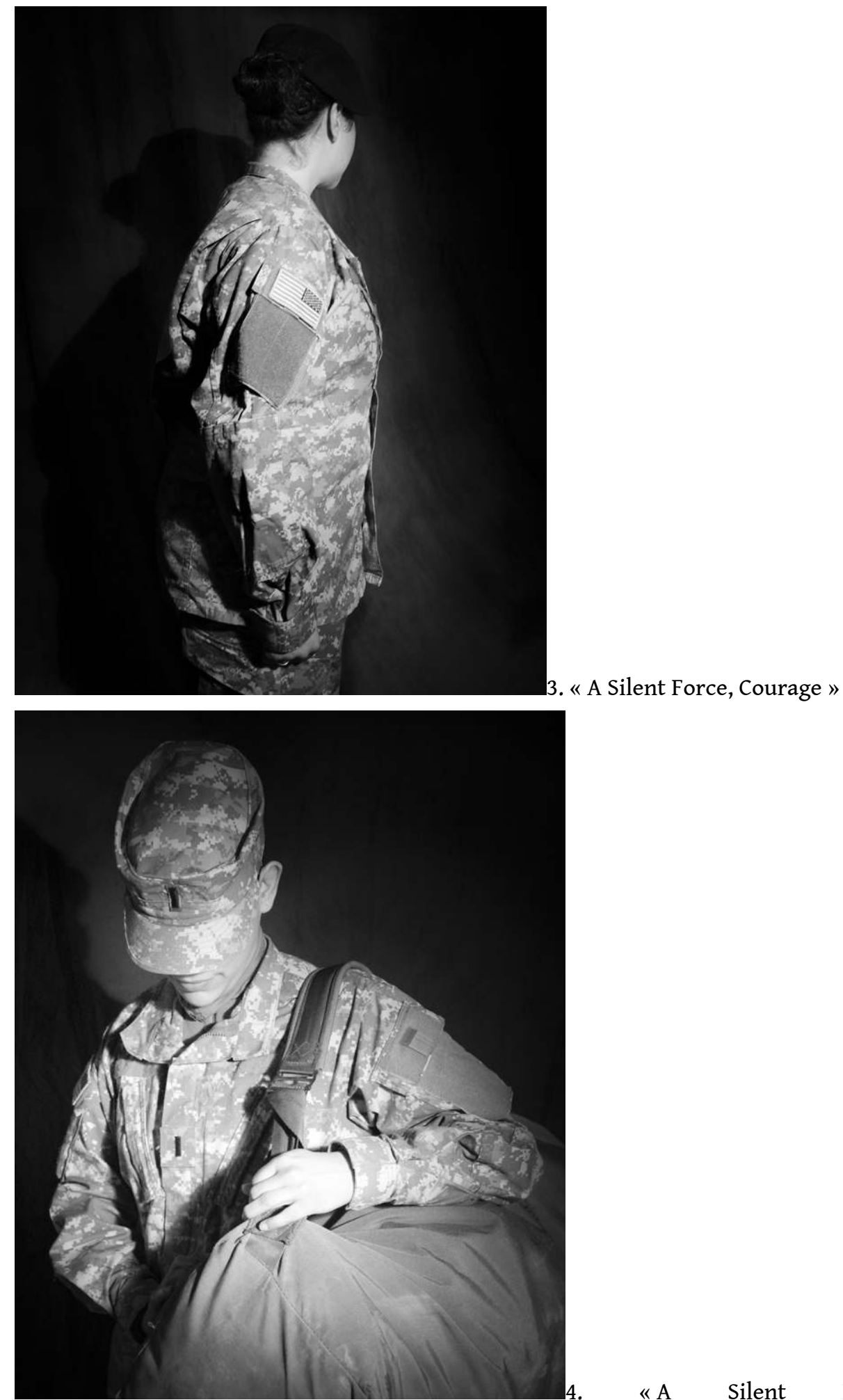

« A Silent Force,

Commitment » 


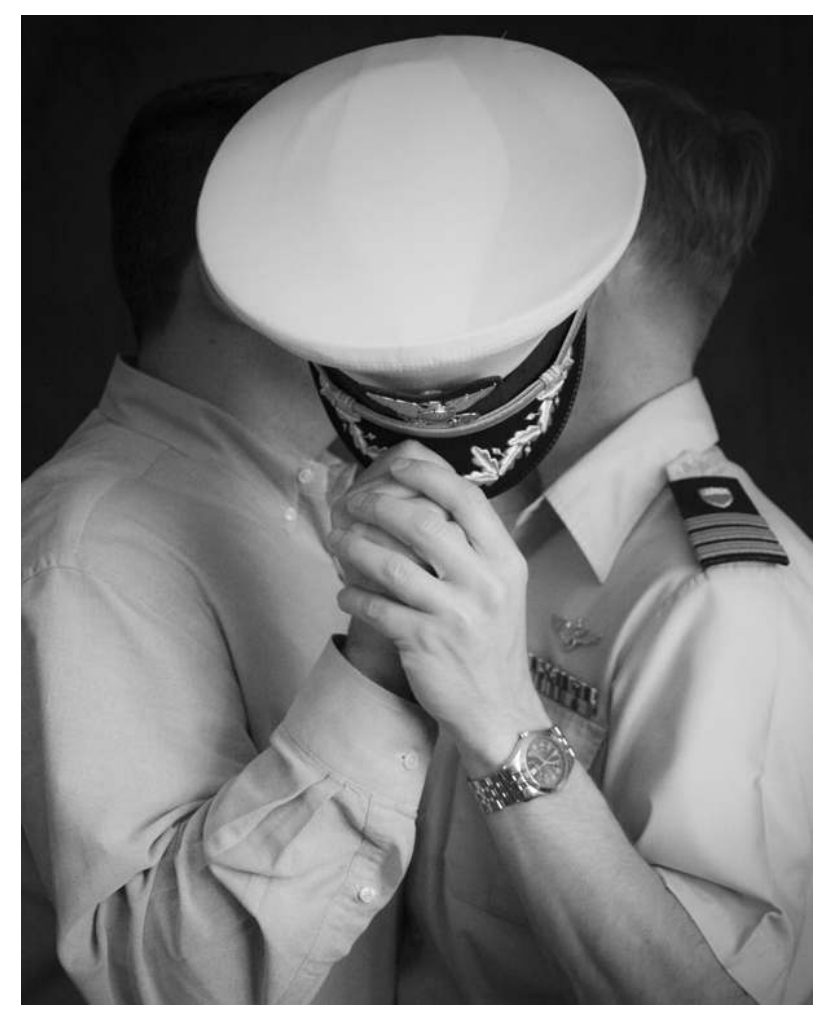

Les photographies d'Anthony Loverde montrent des militaires qui tournent le dos ou cachent leur visage par différents moyens. Comme chez Jeff sheng, ce choix est une façon de mettre en avant aussi le statut d'hommes et de femmes dont l'identité disparaît derrière l'institution militaire. Pourtant, on sent chez les sujets d'Anthony Loverde comme un désir de soulever la chape de plomb qui accable le militaire obligé de taire son identité sexuelle et qui passe notamment par la revendication du droit constitutionnel.

L’image suivante, « A Silent Force, Selfless Service » est à cet égard exemplaire. 


\section{5. "A Silent Force, Selfless Service »}

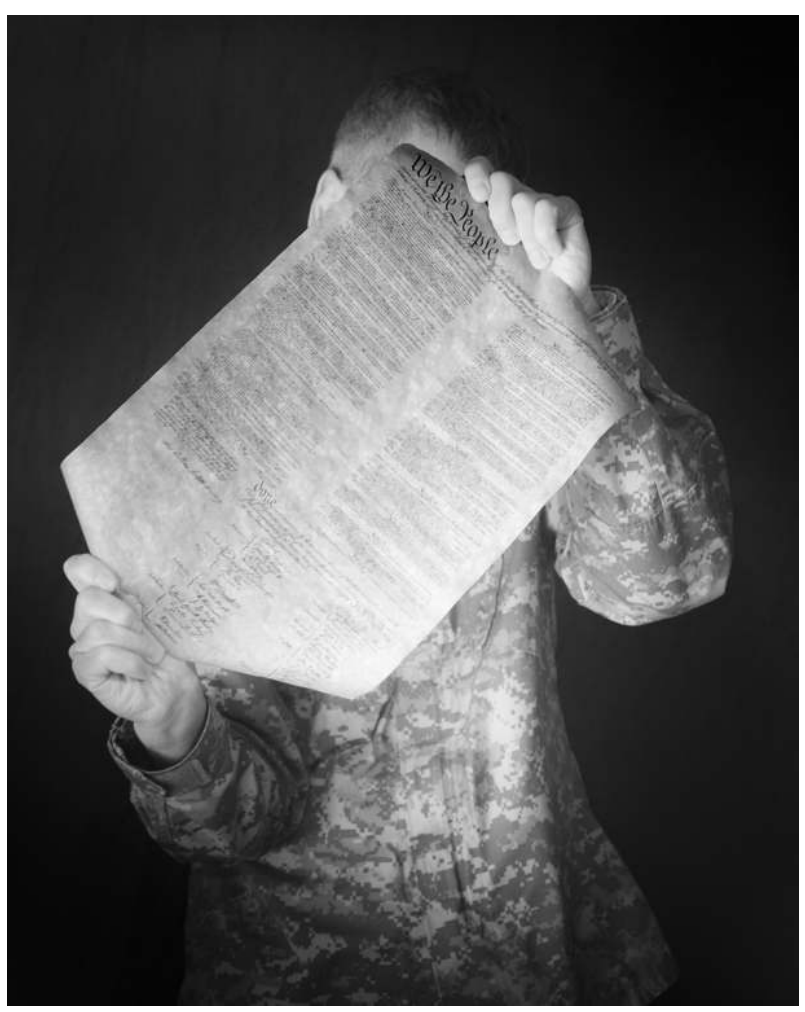

Cette photographie représente un homme qui brandit la Constitution américaine, où se distinguent les célèbres mots d'ouverture : «We The People ». Cet homme en uniforme incarne l'institution éminemment respectée qui forge les héros de la nation et le service rendu à la patrie. Pourtant, la Constitution, qui apparait ici au premier plan, fait figure de bouclier derrière lequel il protège son visage.

L'œil du spectateur cherche le regard du sujet photographié. Il est possible que le militaire soit en train de lire une inscription, ou peut-être ses droits ? - au dos du parchemin qu'il tient à hauteur d'homme, face à l'objectif du photographe, c'est-à-dire à la hauteur du regard du spectateur, en lieu et place de son propre visage. Dans le texte constitutionnel où est revendiquée la force inaliénable du droit, il est question d'identité, non pas sexuelle, mais citoyenne, renvoyant à celle qu'ont voulue les pères de la nation. Il semble que le militaire, dont on discerne à peine le contour du visage, cherche sa place dans le cadre qui est moins celui de la photographie que celui que forme le parchemin.

Si son geste ne semble pas véritablement revendicateur, c'est probablement qu'il se veut le simple rappel d'une vérité fondamentale, qui est celle d'une identité partagée avec tous les Américains.

La référence à la Constitution n'est bien sûr pas un hasard. Comme d'autres minorités avant les homosexuels, les Noirs ont milité pour l'égalité des droits pendant les années 1960 en invoquant la Constitution. Aubrey Sarvis, directeur exécutif du Servicemembers Legal Defense Network (SLDN), le réseau de défense juridique du personnel militaire, a ainsi écrit dans un éditorial publié sur le site Internet de l'organisation, au sujet de la journée du 22 décembre 2010 : «J'ai pu dire au Président Obama que c'était sa journée Lyndon Johnson et qu'elle ferait date. Sans l'ombre d'un doute, il s'agit là du texte de loi décisif de sa génération en matière de droits civiques ${ }^{5}$. 
Suivant la volonté du pouvoir exécutif de passer devant le Congrès, la Chambre des Représentants a d'abord entériné l'abrogation du texte de loi le 15 décembre 2010. Puis, ce fut au tour du Sénat, le 18 décembre, notamment grâce aux voix de huit sénateurs républicains qui ont voté avec les démocrates.

Pour nombre d'associations de soutien aux droits des homosexuels, l'abolition de «Don't Ask, Don't Tell» est une nouvelle étape vers la légalisation du mariage entre personnes de même sexe. Cette fois, la bataille se livre au niveau fédéral. Le procès qui s'est ouvert le 12 janvier devant une Cour fédérale de San Francisco (Californie) s'annonce décisif. Actuellement, cinq états américains autorisent les unions homosexuelles: l'Iowa, le Connecticut, le Massachusetts, le Vermont et le New Hampshire. La bataille juridique en Californie oppose partisans et opposants à la Proposition 8. Adoptée par référendum, cette proposition interdit les unions entre personnes de même sexe et annule une décision de la Cour suprême de Californie en faveur de ces mêmes unions. Le dossier pourrait ainsi finir devant la Cour suprême des États-Unis, dont la décision scellerait de manière définitive le sort des unions homosexuelles sur l'ensemble du territoire américain, sans possibilité de recours pour l'une ou l'autre des parties.

À travers leur travail, Anthony Loverde et Jeff Sheng décrivent tous deux des sujets dont l'identité a été effacée, ou du moins fortement affectée par cette loi. Cette partie d'eux-mêmes qu'une institution tente de dissoudre, ils lui donnent, paradoxalement, un nouveau visage, à travers une représentation de l'intime chez Sheng, par le biais de l'amorce d'un mouvement revendicatif des droits constitutionnels chez Loverde. Tous les deux expriment l'urgence à pouvoir vivre leur homosexualité à visage découvert. Dès le texte de loi abrogé, Jeff Sheng a annoncé qu'il allait donner suite à sa série de photographies.

\section{BIBLIOGRAPHIE}

\section{CREDITS PHOTOGRAPHIQUES :}

\section{Jeff Sheng}

Toutes les photographies de Jeff Sheng ont été reproduites avec la gracieuse permission de l'artiste et de la galerie Kaycee Olsen Gallery, Los Angeles.

« Mike, Boston, Massachusetts, $2009 »$

« Jess, Bend, Oregon, $2009 »$

« Grace, Fort Jackson, South Carolina, 2010 »

« Katherine \& Elizabeth, Denver, Colorado, $2010 »$

« Dean, Montgomery, Alabama, $2010 »$ 
Pour plus d'informations:

www.jeffsheng.com

www.kayceeolsen.com

\section{Anthony Loverde}

Toutes les photographies d'Anthony Loverde datent de 2009 et ont été reproduites avec la gracieuse permission de l'artiste.

"A Silent Force, Integrity »

«A Silent Force, Honor »

«A Silent Force, Courage »

"A Silent Force, Commitment »

« A Silent Force, Selfless Service »

\section{NOTES}

1. Jeff Sheng (2010). Fearless Portfolio, (Self-published by Jeff Sheng and The Kaycee Olsen Gallery in Los Angeles), http://www.kayceeolsen.com/artist/seriesview/1926/409

2. Jeff Sheng (2010), Don't Ask, Don't Tell: Volume 1, (Self-published by Jeff Sheng and The Kaycee Olsen Gallery in Los Angeles).

Jeff Sheng (2010), Don't Ask Don't Tell: Volume 2, (Self-published by Jeff Sheng and The Kaycee Olsen Gallery in Los Angeles).

3. Anthony Loverde, A Silent Force: Men and Women Serving Under Don't Ask, Don't Tell, Bloomington, AuthorHouse, 2010

4. Échange de courriel avec l'auteur, 14 octobre 2010.

5. Aubrey Sarvis, "Frontlines feed: The Latest on Repealing "Don't Ask, Don't Tell": Letter from the executive director, SLDN December Newsletter," Servicemembers Legal Defense Network, December 2010, http://www.sldn.org/blog/archives/sldn-newsletter-july-2010/

INDEX

Thèmes : Trans'Arts

\section{AUTEUR}

\section{CLÉMENT MASSÉ}

Journaliste, correspondant France 24 à Los Angeles 